\title{
Central Monitor Based On Personal Computer Using Single Wireless Receiver
} (SPO2 Parameter)

\author{
Ahmad Fanani, Bambang Guruh Irianto, Andjar Pudji \\ Department of Electromedical Engineering Poltekkes Kemenkes Surabaya \\ Surabaya, Indonesia \\ apanpanani@gmail.com,bgi_ps@yahoo.com,andjar.pudji@gmail.com
}

\begin{abstract}
Central monitor is a tool that monitors the patient's condition with several devices into one display on a personal computer (PC). Pulse Oximetry serves to monitor the state of oxygen saturation in the patient's blood without going through blood gas analysis. This tool uses a wireless delivery system, HC-11 that can transmit data as far as 10 meters without obstruction. This tool uses a finger sensor, an analog signal conditioner and a microcontroller that is processed to produce a percentage value of $\mathrm{SpO} 2$ which is then sent through $\mathrm{HC}-11$. The workings of this tool are very simple by entering the finger sensor on the finger and then it will be detected by the finger sensor that will be displayed on the PC. Digital data from the ADC Atmega is received by the personal computer (PC) via Bluetooth HC-011. Furthermore, the data is processed with the Delphi program and displayed on the monitor. Appears on the Delphi application. After measurement, get an error in the SpO2 parameter, the biggest error is $1.02 \%$ and get the smallest error $0.8 \%$.
\end{abstract}

Keywords: finger sensor, HC-11.

\section{INTRODUCTION}

In every hospital, there are always patient patients to help the performance of doctors and nurses. Patient monitors are medical devices that are used to monitor patients' physiological conditions. Patient monitors are standard examinations, namely ECG, respiration, blood pressure or NIBP, and blood oxygen levels or blood saturation or $\mathrm{SpO} 2$ [1]. The alarm serves as an indicator to remind health workers if there is a decrease in oxygen saturation below the level of $90 \%$ and a low pulse rate. Adding an alarm will add value to the use of pulse oximetry that is more automatic and responds quickly to patient safety. By using a buzzer circuit that is connected to the microcontroller, alarm parameters can be set properly [2]. If the human body lacks or excess oxygen, it will cause illness and disruption to other body's work systems. Some diseases caused due to lack or excess oxygen include hypoxemia, anemia, and so on. At some level, the disease can pose a risk of death [3]. Lack of oxygen supply in the body can cause tissue damage to the body due to hypoxia [4].

Based on the results of a library search from Pulse Oximeter that was made by Teguh Pratomo (2016) about "Pulse Oximeter Fingertip PC Display (SpO2)", it was mentioned that the Tool was not equipped with storage for Pleth's signal analysis process and needed software improvements so that the display was automatically set according to the reference of blood concentrations of each patient. A tool "Designing Oxygen Saturation Measuring Instrument in LCD Graph Blood Display" was made by Pramita Galuh Ajeng Pradana in 2017, where this tool has been able to display signals on LCD charts but the programming used is still Avr and the tool cannot be used for central monitoring.

Then in 2014, research was developed by Desak Putri Puspita Indriani, Yudianingsih, Evrita Lusiana Utari entitled "Designing Pulse Oximetry with Priority Alarms as a Vital Monitoring for Patients" This study still has an oxygen saturation measurement value that is not accurate because the components used are still ordinary components not from tantalum. Research has been carried out with the title "Monitoring Heart Rate equipped with Temperature Sensors by Sending Data to PC via Bluetooth" by Duta, 2016. In this study using the Atmega 8535 microcontroller as a data collector and displayed on a PC using wireless. To determine the temperature of the patient using LM35 and the BPM value of the patient obtained from processing a series of BPM instrumentation that uses a finger sensor (sensor on the finger). This tool also features an internal PC storage with patient data. But the tool is still designed to monitor one module in patients.

Based on the results of the identification of the problems above, the author will make a Central Monitor based on a Personal Computer (PC) Via Wireless with 1 Receiver (SpO2). Where this tool uses 1 receiver to save in the cost of the manufacturing process. With the hope that monitoring can be done through a wireless system or without using cable media as data transmission.as a medium for sending and displaying data to overcome the shortcomings of previous studies. 


\section{MATERIALS AND METHODS}

\section{A. Experimental Setup}

This study was applied to patients over the age of 17 years. Data retrieval is done on random respondents 5 times and data is displayed in real-time with a measurement time of 7 seconds alternating between module 1 and module 2. Data collection is carried out at a distance of 5 meters and 10 meters without any obstacles. Data collection was carried out with 2 patients simultaneously. parameters used by $\mathrm{SpO} 2$.

\section{1) Materials and Tool}

This study uses the Nellcor brand finger sensor for oxygen saturation parameters in the body. Finger sensor is attached to the patient's finger. The component used uses the Arduino Nano as a Microcontroller to provide $\mathrm{SpO} 2$ data obtained from the finger sensor connected to the HC-11 Bluetooth module port to send data and display on the Personal Computer.

\section{2) Experiment}

In this study, the researchers compiled a module that conditions the signal for oxygen saturation in the body from a finger sensor. Data is sent with a Bluetooth delivery system using the HC-11 module. The results will be displayed on a PC using the Delphi application. In seven second the results will be sent alternately between module 1 and module 2 . The researcher conducted several tests including:

a. Test for processing oxygen saturation data using an automatic reference system.

b. Comparison of module measurement results used for $\mathrm{SpO} 2$ parameters using a pulse oximeter.

c. comparison of results with a distance of 5 meters and 10 meters

\section{B. Diagram Blok and Flowchart}

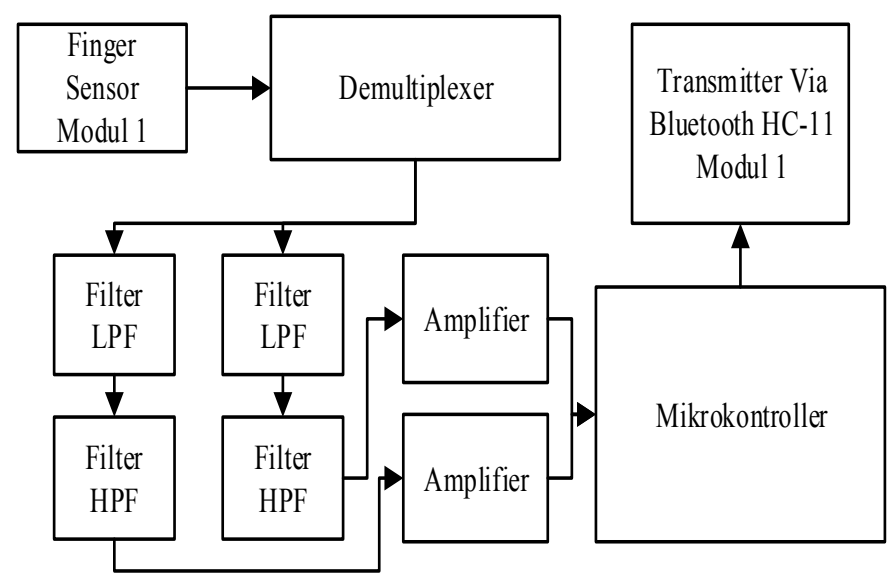

Fig 1. The SpO2 circuit diagram block

The index finger of the patient is fitted with a finger sensor which serves to detect oxygen saturation signals in the blood, then the signal will be processed in a PSA circuit consisting of
HPF and LPF filters and amplifiers that are amplified 101 times so that the signal detected by the finger sensor can be clearly seen and ready to be sent to the ADC port on the microcontroller as analog data input. Then processed into digital data and data sent using Bluetooth $\mathrm{HC}-11$ then displayed on a PC in the form of a percentage value of oxygen levels. Then it is received by the receiver circuit and processed through a PC. When the oxygen level is less than the normal value, the alarm will sound as a warning.

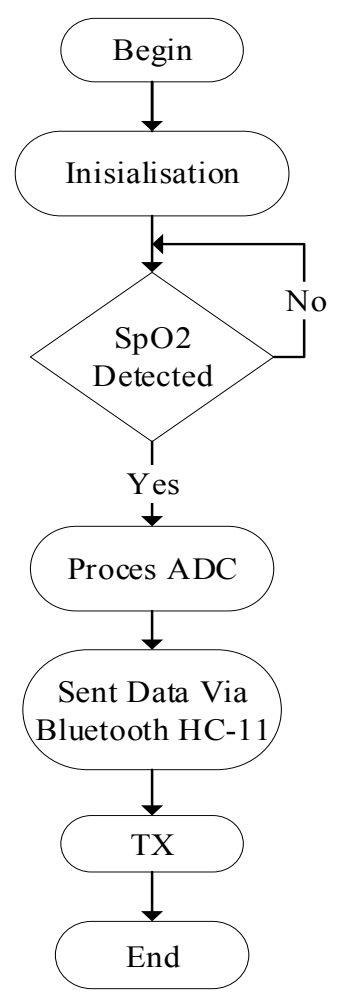

Fig 2. Saturation oksigen and transmitter Flowchart

The digital finger sensor reading reading starts with reading the ADC as a result of processing analog signals on the finger sensor. flow starts from the read sensor sent in variable and sent to the HC-11 Bluetooth module. The results of AC and DC from RED and IR enter the ADC A3, A4, A6, and A7 pins. The SpO2 signal entering the ADC pin will be converted by the Minimum System into digital data. Then from digital data then it will be processed and transmitted with the $\mathrm{HC}-11$ wireless module. The data is then received by the Receiver using the $\mathrm{HC}-11$ module connected with the PL 2303 module connected to the personal computer so that the information received as string data will be processed using the Delphi 7 application. 


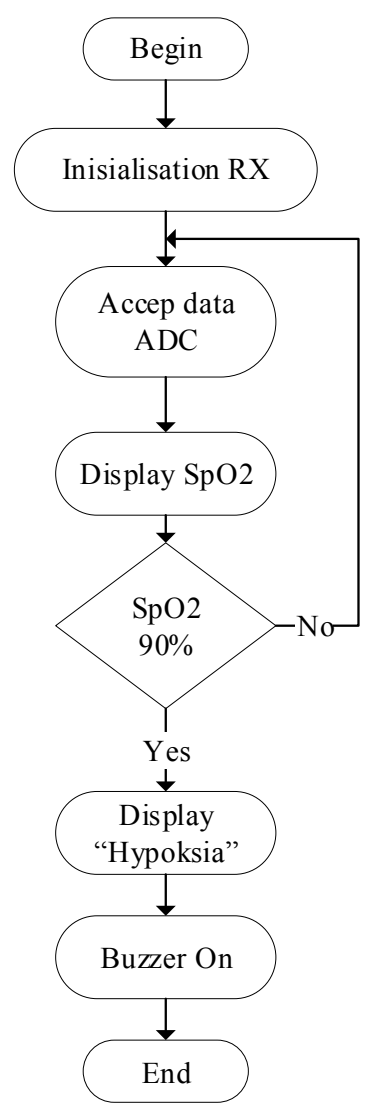

Fig 3. Receiver Flowchart

The reading flow rate begins by reading the ADC as a result of the agreement of the analog signal on the finger sensor. The ADC signal is processed and calculated as the results displayed on the PC.

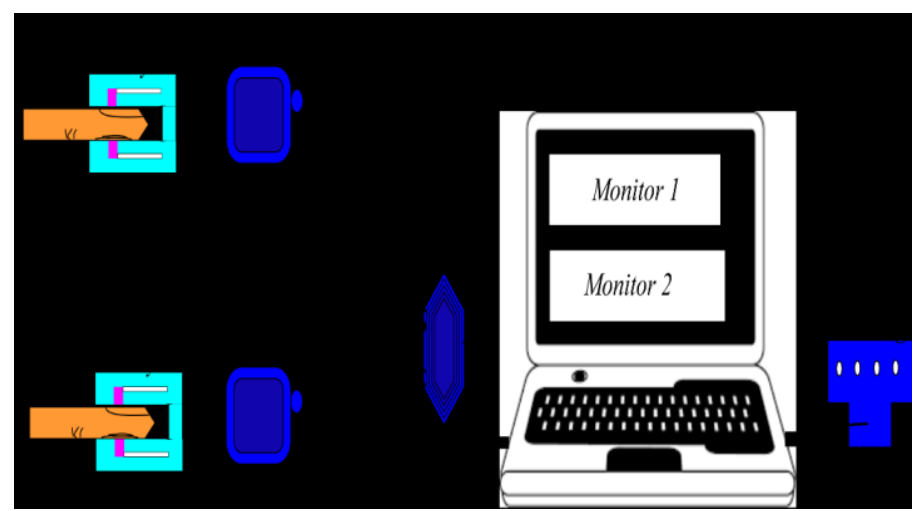

Fig 4. Mechanism diagram

Module 1 and module 2 data is sent via the HC-11 Bluetooth module where there is only one receiver. displayed on the PC and there is an alarm warning if there are incorrect parameters.
C. Circuit

\section{1) Finger Sensor Circuit}

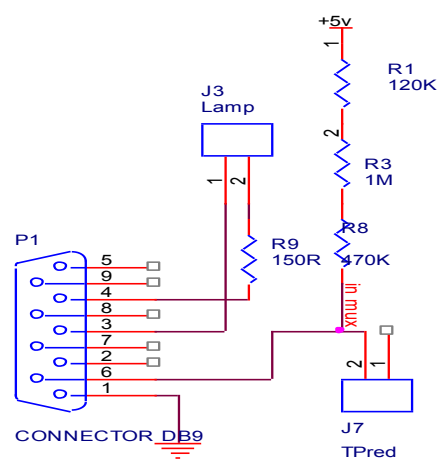

Fig 5. Finger Sensor Circuit

The sensor circuit above works with a voltage divider system. Photodiodes receive continuous light from infrared and then processed into voltage.

2) Demultiplexer Circuit

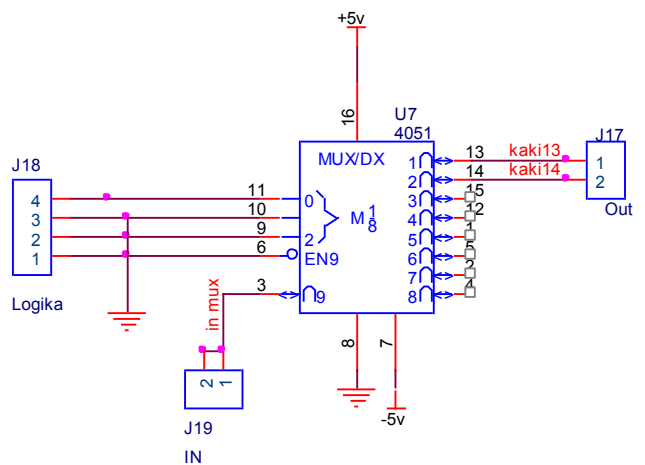

Fig 6. Demultiplexer circuit

This is the measurement result on the $1199 \mathrm{~Hz}$ Demultiplexer Input, $584 \mathrm{mV}$ amplitude with the sensor installed in the patient obtained from the photodiode output, the AC and DC signal demultiplexer input from RED and IR are still mixed.

3) Demultiplexer Circuit

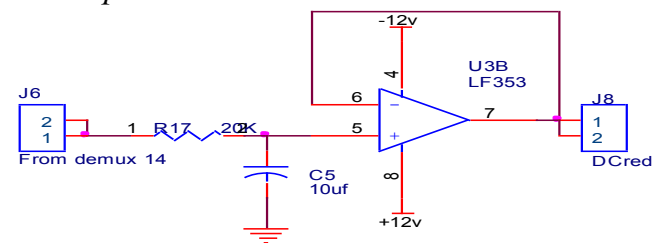

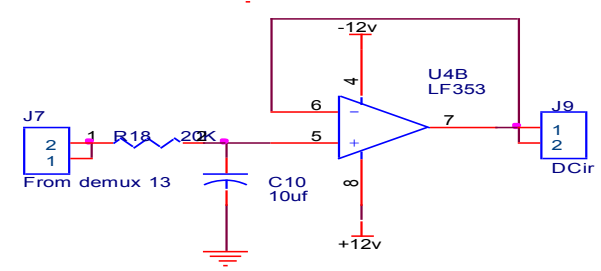

Fig 7. Filter LPF 
This is the measurement result on the output amplifier and the second filter of the Frequent 14th Demultiplexer foot, $609.7 \mathrm{mHz}$, the amplitude is $6.60 \mathrm{~V}$. This is the second gain of the AC RED signal, which is amplified again by a gain of $101 \mathrm{X}$ after the first gain. $1 \mathrm{KHz}$ noise artifacts look thinner because filters are used with the same cut-off frequency so the filter presses better.

$$
\begin{aligned}
\text { F cutoff } & =\left(\frac{1}{2 P i . R f . C f}\right) \\
\text { Acl } & =1+\left(\frac{R F}{\operatorname{Rin}}\right)
\end{aligned}
$$

\section{4) Demultiplexer Circuit}

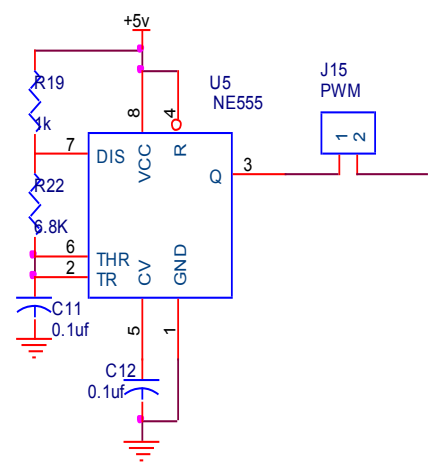

Fig 8. PWM circuit

This is the measurement result on the $1199 \mathrm{~Hz}$ Demultiplexer Input, $584 \mathrm{mV}$ amplitude with the sensor installed in the patient obtained from the photodiode output, the $\mathrm{AC}$ and DC signal demultiplexer input from RED and IR are still mixed.

\section{RESULTS AND DISCUSSION}

In this study, comparison of data results with predetermined comparison tools, measurement, and analysis of delivery times, and calculation and analysis of lost shipping data were conducted.

\section{1) Heartbeat Calculation Using Auto Reference Result}

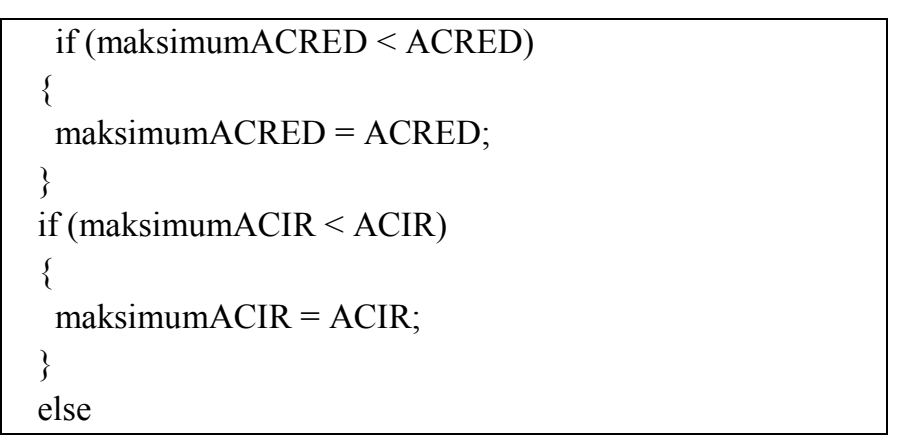

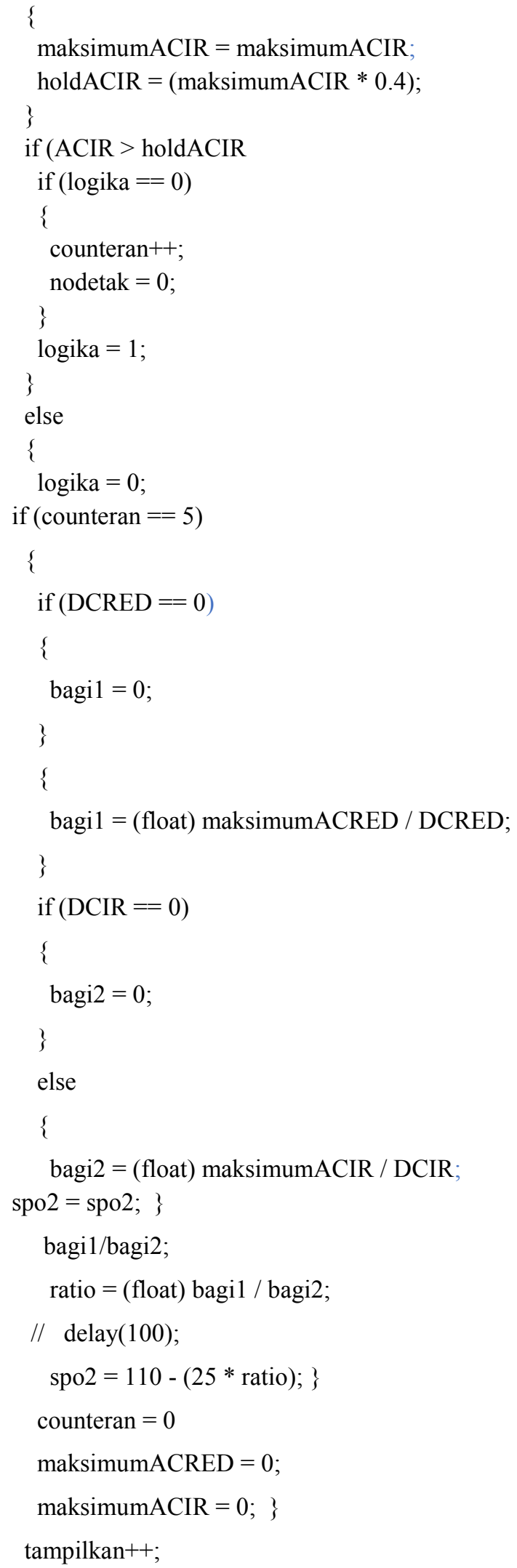




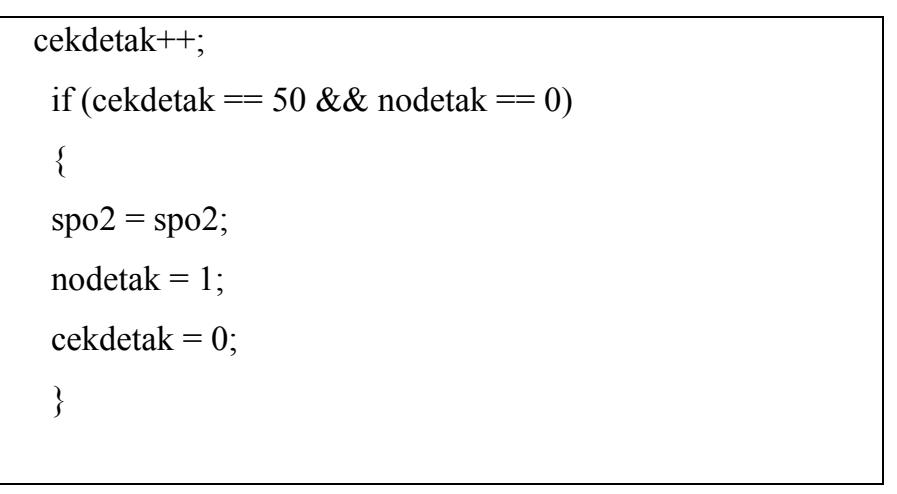

The data processing program listing on Arduino starts from reading the $\mathrm{ADC}$ through pin converting $\mathrm{ADC}$ data to voltage. In the above program, listings use an auto reference system that uses the highest percentage of signal voltage to result $\mathrm{SpO} 2$.

\section{2) Result of the $\mathrm{SpO} 2$ and Pulse Oximetry}

TABLE I. MEASUREMENT ERROR BETWEEN MODULE AND COMPARISON APPARATUS

\begin{tabular}{ccc}
\hline No & Subject & Error SpO2(\%) \\
\hline $\mathbf{1}$ & P1 & 0.2 \\
\hline $\mathbf{2}$ & P2 & 0.4 \\
\hline $\mathbf{3}$ & P3 & 0.82 \\
\hline $\mathbf{4}$ & P4 & 1.03 \\
\hline $\mathbf{5}$ & P5 & 1.02 \\
\hline
\end{tabular}

Based on the results of the measurement of the $\mathrm{SpO} 2$ value on this central monitor module it has an error value of $1.03 \%$. From this value, it can be seen that this module is said to be used because the error value does not exceed the maximum limit value of $\pm 1 \%$.

\section{3) Modul Result}

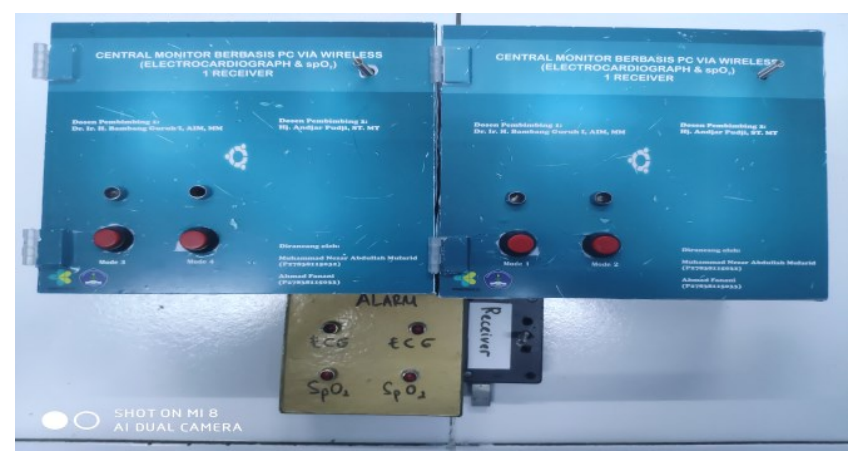

Fig 9. The design of the central monitor
The picture above shows a central monitor module consisting of 2 parameters 1 receiver modules and an alarm warning.

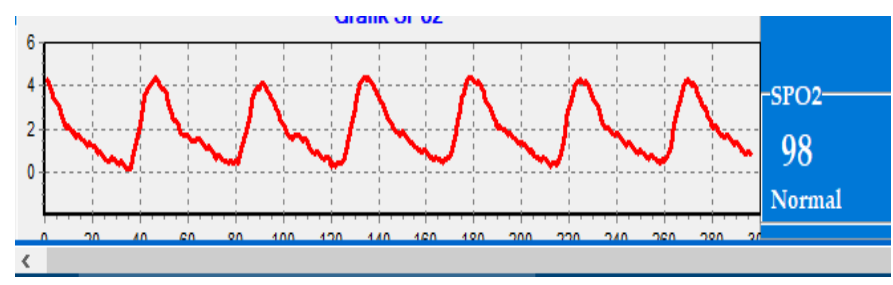

Fig 10. Display Signal SpO2

SpO2 display signal and value displayed on the PC using the Delphi application. In the picture shows the number 98 and the normal indication.

\section{DISCUSSION}

After comparing the results and measurements of the instruments, the average error in the $\mathrm{SpO} 2$ parameter is $0.7 \%$ of the temperature value $\pm 1{ }^{\circ} \mathrm{C}$. The test results of missing data with a distance of 10 meters $1 \%$ error displayed on the PC using the Delphi application and on the signal there is noise even though it is not too large. The results of testing the delivery time with a distance comparison, it can be concluded that sending using Bluetooth $\mathrm{HC}-11$ can send data as far as 10 meters. for the development of data can be improved in the delivery process

\section{CONCLUSION}

This research shows that central monitors are monitoring vital signs that are carried out in real-time and alternately. This technology uses sending via Bluetooth HC-11 can be applied properly. this allows the maintenance monitoring process without cable media and makes it easy for technicians to troubleshoot. The design of this tool is to maximize the process of monitoring outpatient conditions.

\section{REFERENCE}

[1] A. H. Noviyanto, "PERANCANGAN KEAMANAN DATA PADA DATA HASIL MONITORING PASIEN," pp. 38-42, 2014.

[2] D. Putri, P. Indriani, and E. L. Utari, "Perancangan Pulse Oximetry Dengan Sistem Alarm Prioritas Sebagai Vital Monitoring," pp. 93-107, 2014.

[3] S. Khairunnisa, I. D. Gede, H. Wisana, I. Priyambada, C. Nugraha, and J. T. Elektromedik, "Rancang Bangun Pulse Oximeter Berbasis Iot ( Internet of Things )," 2018.

[4] A. Kaunang, R. Wilar, and J. Rompis, "Perbandingan Kadar Saturasi Oksigen Hari Pertama," J. e-Clinic, vol. 3, no. April, pp. 397-401, 2015. 